\title{
Pre-fatigue influence on quasi-static tensile properties of Ti-6Al-4V in thin-sheet form
}

\author{
J. Galán López ${ }^{\mathrm{a}}$, P. Verleysen, I. De Baere, and J. Degrieck \\ Department of Materials Science and Engineering, Faculty of Engineering, Ghent University \\ Sint-Pietersnieuwstraat 41, 9000 Gent, Belgium
}

\begin{abstract}
The response of engineering structures to loads is most often assessed without taking possible damage of the used material(s) into account. However, it has already been proved that a preceding cyclic loading and the alteration of the microstructure it causes, can have a significant influence on the mechanical properties of steel grades and aluminium alloys , and hence on the behaviour of structural elements made of it. Ti-6Al$4 \mathrm{~V}$, the most widely used titanium alloy, is often one of the materials chosen for cyclic loading applications where other solicitations are present too. Therefore, the influence of pre-fatigue on the quasi-static mechanical properties of Ti-6Al-4V in thin-sheet form is investigated. Tensile experiments are performed on samples subjected to different damage levels. The material does not show a clear dependence of its tensile properties with previous loading cycles, although the overall effect can be important for particular geometries.
\end{abstract}

\section{Introduction}

Some materials, subjected to a cyclic load, eventually fail by a sudden fracture. Therefore, the loading cycles are inducing a permanent damage in the material. Fatigue theories study the accumulation of this damage in order to predict the number of cycles at which final failure will happen. However, it is not uncommon that mechanical parts subjected to cyclic loading have to support not only the cyclic loads but also relatively high static loads, or even impact events. These loads are applied on damaged material, but before its final failure (this state of the material will be referred as "pre-fatigued" in the following sections). Therefore, the influence of fatigue damage on other mechanical properties has to be known in order to assess the real response of the material.

Previous work has shown the importance of pre-fatigue in the impact response of high strength steels: at half of their fatigue life their energy absorbing potential is already severely degraded [1]. Studies on aluminium alloys [2] have concluded that the tensile behaviour does not only depends on the prior fatigue loading conditions. Instead, the effect of previous fatigue is strongly linked to the material's microstructure. In particular, experiments show that some aluminium alloys are not affected at all by previous fatigue, whereas other alloys of the same material have a strong dependence.

The influence of pre-fatigue in the behaviour of steels and its lack of effect on some aluminium alloys have been confirmed by further studies [3]. Additional results from quasi-static experiments indicate that pre-fatigue can have a different effect on the tensile response of the material depending on the strain rate. Other quasi-static tensile experiments performed in previously fatigued steel also show evidence of mechanical properties degradation along the fatigue life of the material [4]. Observations in SEM and TEM revealed the importance of dislocations in this process.

Ti-6Al-4V is a multi-phase material. It consists of a harder (and richer) $\alpha$ phase which gives it its strength and a softer $\beta$ phase which provides workability. The alloy elements, aluminium $(6 \%)$ and vanadium (4\%), ensure the right balance between both phases and provides lighter weight ( $\mathrm{Al}$ ) and

a e-mail: Jesus.GalanLopez@UGent.be

This is an Open Access article distributed under the terms of the Creative Commons Attribution-Noncommercial License 3.0, which permits unrestricted use, distribution, and reproduction in any noncommercial medium, provided the original work is properly cited. 
better formability (V). This titanium alloy has low weight and good mechanical properties (in particular high strength and fatigue life) and is resistant to hostile service conditions as high temperatures and corrosive environments. It is widely used in the industry and well known by the scientific community. The effect of pre-fatigue on the quasi-static tensile properties of Ti-6Al-4V in normal conditions is studied in the present work.

\section{Experimental Procedure}

Next to fatigue experiments to determine the fatigue life at two load levels, quasi-static tensile experiments are performed on as-received and pre-fatigued samples.

All test specimens are obtained by spark erosion from a cold-rolled and annealed sheet of Ti$6 \mathrm{Al}-4 \mathrm{~V}$ with a thickness of $0.6 \mathrm{~mm}$ (manufactured by TIMET $($ ). The same geometry is used for both fatigue and quasi-static tests (figure 1). The gauge length is $50 \mathrm{~mm}$ and the minimum width is $12.5 \mathrm{~mm}$. The dimensions of the grip and transition zones have been chosen so that they have no influence on the load present on the central region of the specimen during the tests. This geometry is a compromise between the limits imposed by ASTM for fatigue testing (ASTM-E466) [5] and quasistatic tensile testing (ASTM-E8[M]) [6]. Situations of disagreement between both standards have been solved relaxing the requirements for fatigue testing, since the original thickness of $0.6 \mathrm{~mm}$ is already rather distanced from commonly used dimensions for fatigue specimens, while tensile testing in thinsheet material is a well established method.

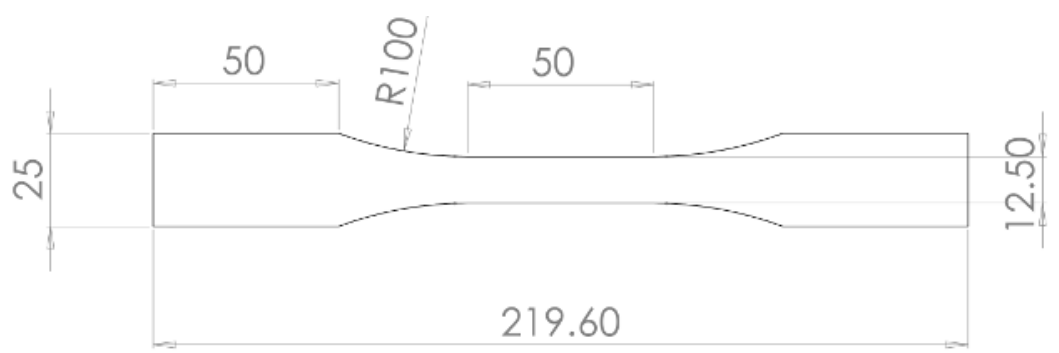

Fig. 1. Specimen geometry (dimensions are given in $\mathrm{mm}$ )

The experiments are performed in an Instron@tensile machine equipped with a dynamic load cell of $100 \mathrm{kN}$. Strain in the specimen is measured with different extensometers depending on the type of loading: static extensometer with a gauge length of $50 \mathrm{~mm}$ for tensile tests and dynamic extensometer with a gauge length of $25 \mathrm{~mm}$ while applying a cyclic load. Strain gauges, glued to some of the specimens, provide additional strain measurements with higher precision at lower strain levels (particularly convenient during cyclic loading at low stress).

In a first stage, three quasi-static tensile tests are performed on damage-free specimens. The speed of testing is imposed in terms of the rate of separation of the two heads of the testing machine, $2 \mathrm{~mm} / \mathrm{min}$, which for the given geometry (gauge length of $50 \mathrm{~mm}$ ) is equivalent to a strain rate of $0.00067 \mathrm{~s}^{-1}$. The mechanical properties of the as-received material, as well as the standard error of the three experiments, are shown in table 1 . The true tensile diagram obtained during one of these tests is included in figures 3 and 4 .

With the aim of determining the loading conditions to apply during pre-fatigue to induce sensible damage levels in the material, fatigue life of six specimens loaded until fracture is determined. Stresscontrolled cyclic loads are applied at the same frequency of $2 \mathrm{~Hz}$ and with a constant stress ratio of 0.1 between the maximum and minimum stress. The frequency is kept low to avoid dynamic effects, while the stress ratio of 0.1 allows a high alternating stress without requiring compression loads (with the consequent buckling of so thin specimens). The maximum cyclic loads applied are 650 and $800 \mathrm{MPa}$, 
in the range $70 \% \mathrm{~S}_{y}-90 \% \mathrm{~S}_{y}$ to avoid plastic deformation. Results of the experiments are summarized on table 2 .

Table 1. Quasi-static mechanical properties of Ti-6Al-4V (three experiments). From left to right: Young modulus, yield stress, tensile strength, uniform elongation, ultimate elongation, absorbed energy at uniform and ultimate strain and area reduction

\begin{tabular}{rrrrrrrrr}
\hline & $\begin{array}{r}E \\
(\mathrm{MPa})\end{array}$ & $\begin{array}{r}S_{y 0.2 \%} \\
(\mathrm{MPa})\end{array}$ & $\begin{array}{r}S_{u} \\
(\mathrm{MPa})\end{array}$ & $\begin{array}{r}E l_{u} \\
(\%)\end{array}$ & $\begin{array}{r}E l_{\max } \\
(\%)\end{array}$ & $\begin{array}{r}U_{u} \\
\left(\mathrm{~J} / \mathrm{mm}^{3}\right)\end{array}$ & $\begin{array}{r}U_{f} \\
\left(\mathrm{~J} / \mathrm{mm}^{3}\right)\end{array}$ & $\begin{array}{r}q \\
(\%)\end{array}$ \\
\hline Mean Value & 117910 & 990 & 1035 & 7.89 & 11.78 & 70 & 94 & 23.12 \\
Error (\%) & 2.62 & 0.61 & 0.72 & 6.95 & 10.81 & 7.85 & 16.20 & 1.13 \\
\hline
\end{tabular}

Table 2. Fatigue life of Ti-6Al-4V $(R=0.1, f=2 \mathrm{~Hz}) . N_{1}, N_{2}$ and $N_{3}$ correspond to experiments under the same conditions, $N_{f}$ is the average of the three values

\begin{tabular}{rrrrrrrr}
\hline $\begin{array}{r}S_{\max } \\
(\mathrm{MPa})\end{array}$ & $\begin{array}{r}S_{\min } \\
(\mathrm{MPa})\end{array}$ & $R$ & $N_{1}$ & $N_{2}$ & $N_{3}$ & $N_{f}$ & $\begin{array}{r}\text { Error } \\
(\%)\end{array}$ \\
\hline 800 & 80 & 0.1 & 7727 & 9193 & 8465 & 8474 & 9 \\
650 & 65 & 0.1 & 24190 & 18000 & 9620 & 17270 & 42 \\
\hline
\end{tabular}

Following mechanical testing, the fracture surfaces of the specimens are observed with Scanning Electron Microscopy (SEM). Metallographic samples of different cross sections, etched with Krolls reagent to reveal their microstructure, are studied with Light Optical Microscopy (LOM). Figure 6 shows micrographs of the central and peripheral zones of the fracture.

In a second stage, quasi-static tests are performed on specimens with three different damage levels, applied under the same loading conditions of the fatigue life tests. In order to determine the number of pre-fatigue cycles to apply, characteristic instants are searched along the life of the material. The evolution of maximum, minimum and medium strain is measured at regular intervals during the fatigue life experiments with the dynamic extensometer (and strain gauges, if present). When the difference of strain at maximum and minimum stress is plotted against the number of cycles (figure 2), three different stages are clearly identified in the graph. Pre-fatigue cycles are applied at maximum load of $800 \mathrm{MPa}$ during 200, 5000 and 2600 cycles (ie. the borders and the middle point of the second stage).

After pre-fatigue, the specimens are subjected to quasi-static tests in the same conditions that asreceived material. True tensile diagrams, next to the diagram of damage free material, are shown in figure 3. Uniform plastic strain is plotted against damage (considering a simple linear Palgrem-Miner law: $D=N / N_{f}$ ) in figure 5. The fracture surfaces are examined with SEM and LOM, and compared with the previous observations.

Results from this first round of pre-fatigue experiments (more extensively discussed in the following section) show that some damaged specimens fail at significantly lower strain levels than asreceived material, but do not show differences in plastic hardening behavior (table 3 and figures 3 and 5). Defects initiated by the cyclic loading, more than changes in microstructure, seem to affect the mechanical behaviour of pre-fatigued material. After inspection with SEM and LOM, fatigue cracks starting at the corners of the sample are identified as the main reason of failure (see figure 7). A new series of experiments are subsequently performed to confirm this hypothesis.

In the last round of experiments $1.5 \mathrm{~mm}$ of material is removed from both sides of the gauge section by spark erosion after the specimens have been pre-fatigued, before performing the tensile test. If small fatigue cracks near the corners are acting as stress concentrators, only once the edges are removed it will be possible to see the real effect of pre-fatigue on the properties of the bulk material. Four sets of three specimens are loaded at two maximum stress levels, 650 and $800 \mathrm{MPa}$, and stress ratio $0.1 \mathrm{up}$ 


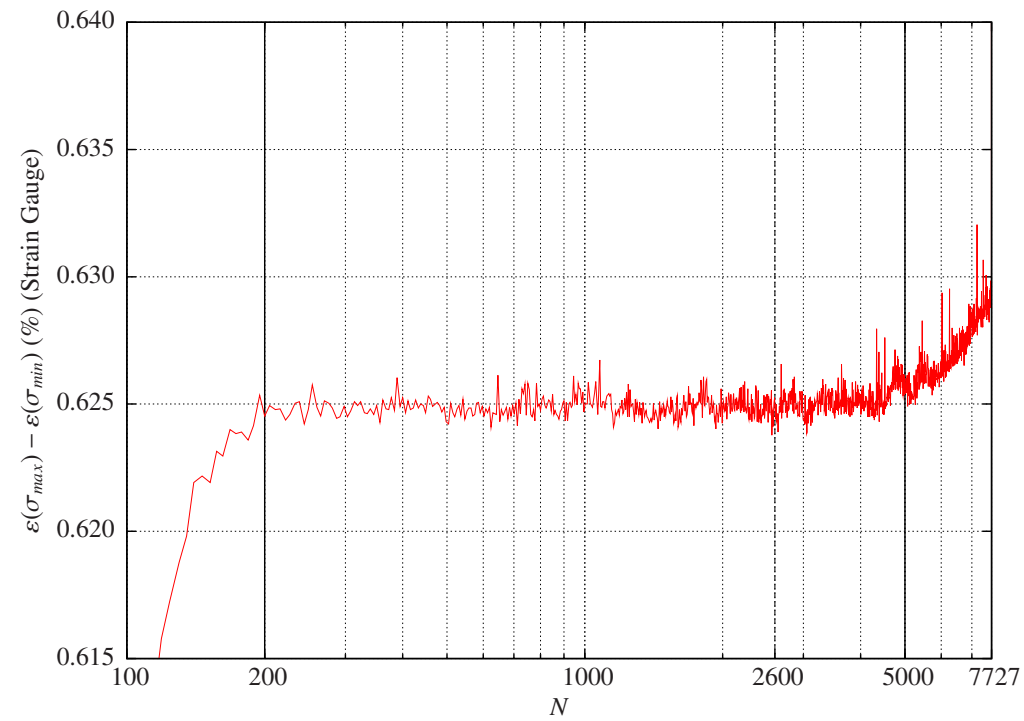

Fig. 2. Evolution of the difference of strain at maximum and minimum stress (800MPa and $80 \mathrm{MPa}$ ) with loading cycles (final life of the specimen: $N_{f}=7727$ ). The strain is measured with a strain gauge attached to the sample. Significant changes in the behaviour of the material are observed after 200 and 5000 cycles

Table 3. Quasi-static mechanical properties of pre-fatigued Ti-6Al-4V. From left to right: number of loading cycles $\left(S_{\max }=800 \mathrm{MPa}, S_{\min }=80 \mathrm{MPa}\right)$, tensile strength, uniform elongation, ultimate elongation, absorbed energy at uniform and ultimate strain and area reduction. Young modulus results - not present in the table - are in the same range that for damage-free material

\begin{tabular}{rrrrrrrr}
\hline$N$ & $\begin{array}{r}S_{y} \\
(\mathrm{MPa})\end{array}$ & $\begin{array}{r}S_{u} \\
(\mathrm{MPa})\end{array}$ & $\begin{array}{r}E l_{u} \\
(\%)\end{array}$ & $\begin{array}{r}E l_{\max } \\
(\%)\end{array}$ & $\begin{array}{r}U_{u} \\
\left(\mathrm{~J} / \mathrm{mm}^{3}\right)\end{array}$ & $\begin{array}{r}U_{f} \\
\left(\mathrm{~J} / \mathrm{mm}^{3}\right)\end{array}$ & $\begin{array}{r}q \\
(\%)\end{array}$ \\
\hline (as-received) 0 & 993 & 1035 & 7.89 & 11.78 & 70 & 94 & 23.12 \\
200 & 998 & 1036 & 7.71 & 12.97 & 71 & 122 & 25.11 \\
200 & 993 & 1034 & 8.12 & 13.11 & 76 & 123 & 22.68 \\
2600 & 984 & 1021 & 7.61 & 11.62 & 68 & 107 & 13.34 \\
2600 & 995 & 1021 & 3.71 & 3.84 & 27 & 28 & 19.37 \\
5000 & 989 & 1029 & 8.10 & 13.20 & 80 & 124 & 3.95 \\
5000 & 991 & 1010 & 2.50 & 2.65 & 15 & 17 & 20.78 \\
5000 & 985 & 987 & 1.00 & 1.06 & 0 & 0 & 8.50 \\
\hline
\end{tabular}

to two different stages of their total lifes. After the previous experiments, 200 cycles are considered a damage level too low to have any effect on the tensile properties of the material, and only 2600 and 5000 cycles are applied at a maximum stress of $800 \mathrm{MPa}$. Additional experiments are performed up to 5000 and 10000 cycles at a maximum stress of $650 \mathrm{MPa}$ (always with $R=0.1$ ) to observe the effect of lower stresses during more cycles. Conditions during fatigue and tensile loading remain being the same than in previous tests. Results are given in figure 4: the tensile diagrams of pre-fatigued samples are not discernible from the one of the as-received material. Uniform strain is independent of the damage level (figure 5).

\section{Discussion}

The quasi-static mechanical properties of Ti-6Al-4V listed in table 1 are in agreement with test results provided by the manufacturer of the material. Fatigue results are not directly comparable with much 


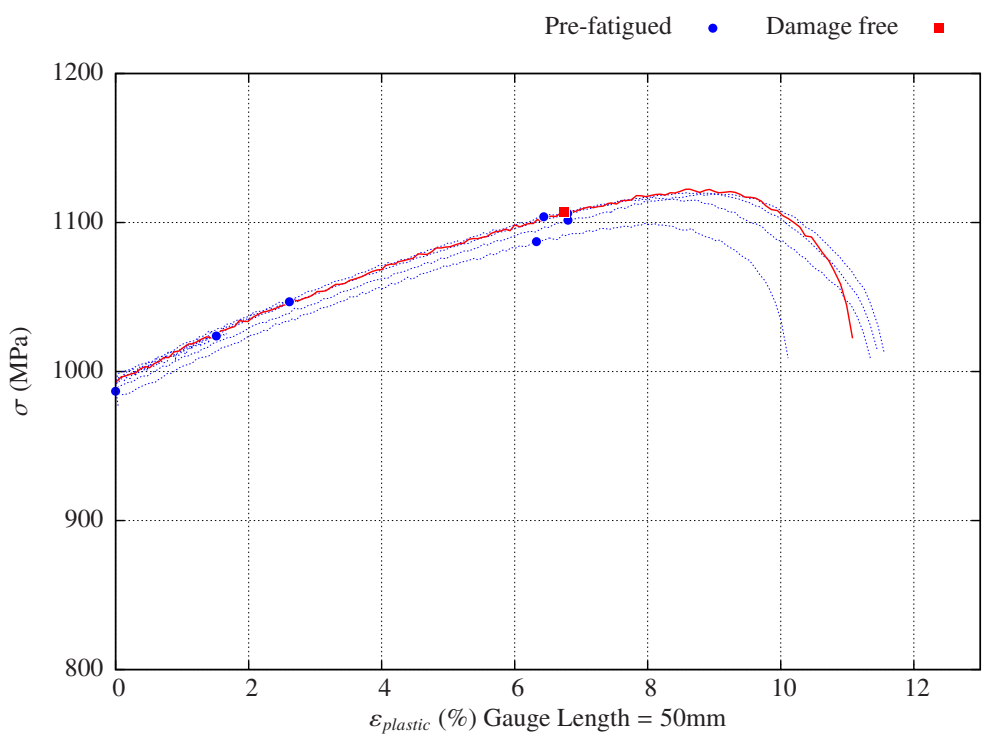

Fig. 3. True tensile curves of the quasi-static tests on as-received and pre-fatigued material. Marks are situated in the point where uniform strain is reached (the point of maximum stress in the engineering diagram or such that $\sigma=\frac{d \sigma}{d \varepsilon}$ using true values). Some pre-fatigued samples fail at lower strain levels following a similar curve

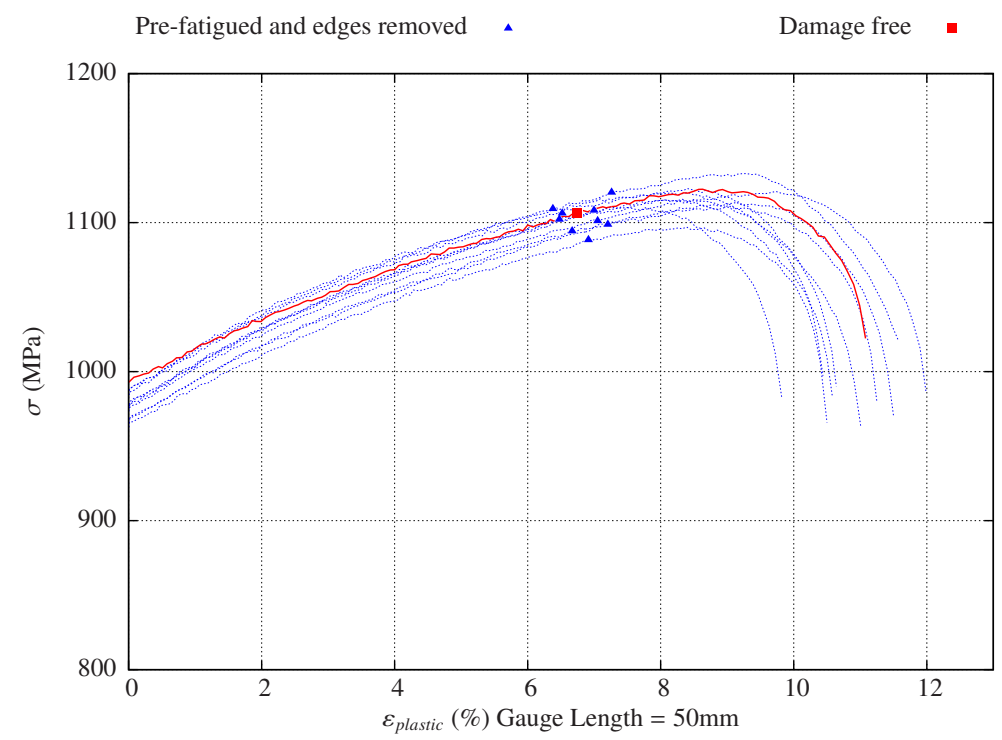

Fig. 4. True tensile curves of the quasi-static tests on as-received and pre-fatigued specimens. After cyclic loading, $1.5 \mathrm{~mm}$ of material are removed from the edges. Marks are situated in the point where uniform strain is reached (as in figure 3). Pre-fatigued specimens behave as damage-free material

of the published data, based on tests performed with specimens of larger cross section. The fatigue failure process of Ti-6Al-4V is dominated by the formation of small cracks around the edges [7] and, since the thickness is only $0.6 \mathrm{~mm}$, a very small crack (even a microscopic one) can behave as an stress concentrator, precipitating the final fracture of the specimen. However, the results are in agreement with published studies based on experiments with similar specimens [8]. 


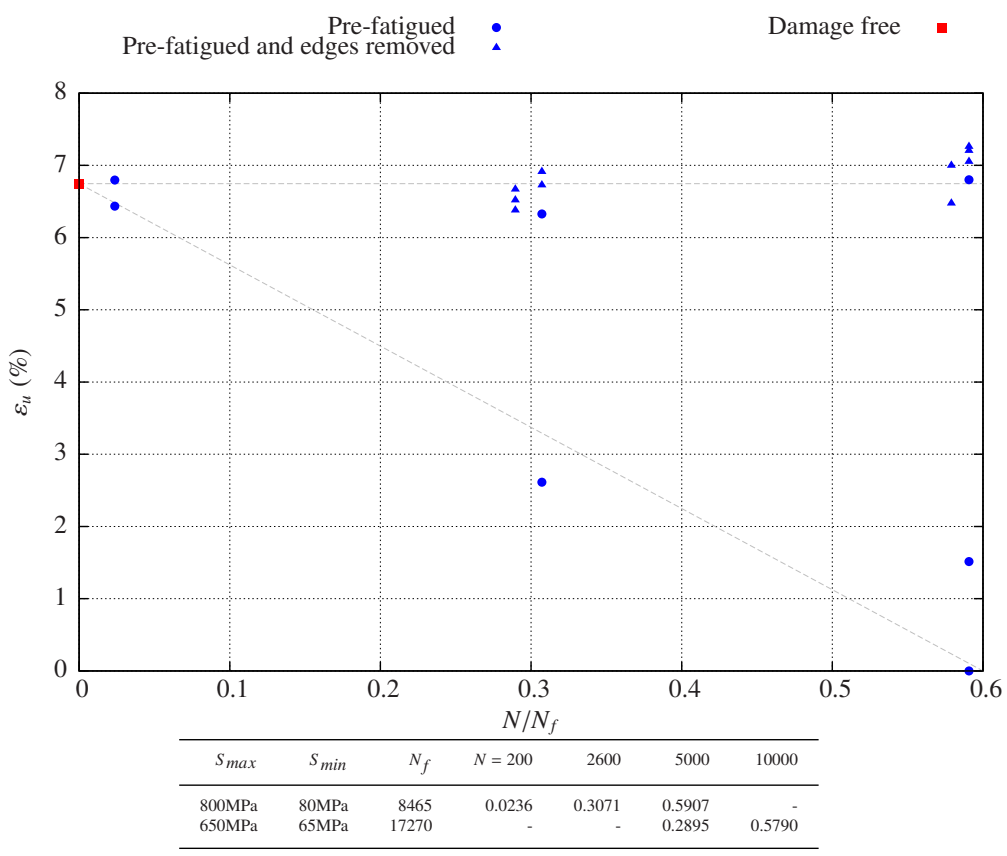

Fig. 5. Effect of pre-fatigue on uniform strain reached during quasi-static tensile testing. The table below the graph indicates the damage level corresponding to the tested loading conditions

Observation of specimens fatigued until the end of their lifes with LOM and SEM disclose the history of the fracture process (figure 6). The most central region of the fracture, where the thickness have been reduced by plastic deformation, presents a ductile fracture. As expected, this region is larger in the samples subjected to higher loads. In contrast, near to the edges of the sample fatigue features can be found, including "beachmarks" (characteristic of interruptions in the propagation periods) and "racket-marks" (perpendicular to the surface from which fatigue fractures originate). Cracks originated in the proximity of the corners of the cross-section are identified as the origin of final failure.

Tensile tests on pre-fatigued specimens follow similar stress-strain curves as those of as-received material, but failure happens at lower values of strain (figures 3 and 5). However, due to the random nature of fatigue, big differences in values are obtained. Since the tensile curves do not show a different behaviour, and SEM micrographs (figure 7) again show fatigue cracks near to the corners and a central ductile fracture, the earlier failure is associated to the same cracks responsible for fatigue failure.

After removing the edges of pre-fatigued specimens, quasi-static test results are comparable to those of as-received material (figures 4 and 5). The fracture surfaces of the specimens do not show any fatigue feature, only a large ductile fracture. There is no evidence of degradation of the mechanical properties because of pre-fatigue.

\section{Conclusion}

The failure process during cyclic loading of Ti- $6 \mathrm{Al}-4 \mathrm{~V}$, for the studied geometry and testing conditions, is dominated by the formation of cracks around the edges of the cross section. These cracks are responsible for the final fracture of the specimen before fatigue damage has a noticeable effect on the static mechanical behaviour of the bulk material. 

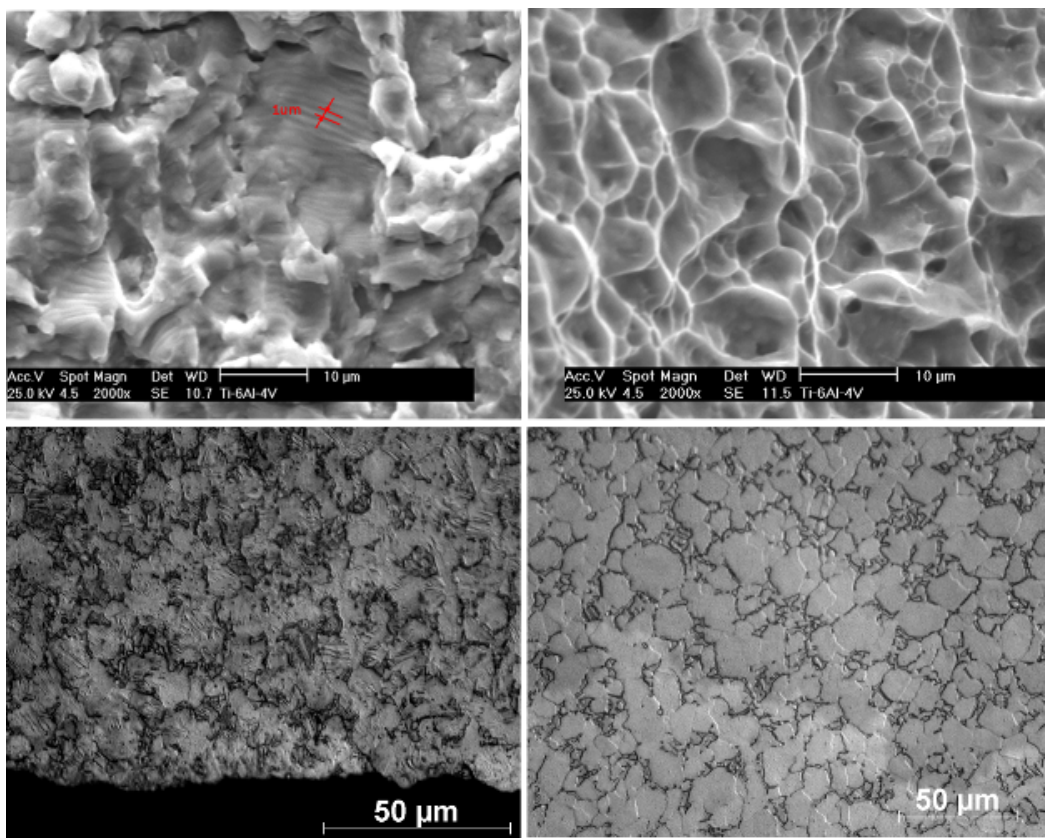

Fig. 6. Top: SEM micrographs taken near to the edges (left) and in the middle of the fracture (right). Bottom: LOM image of fatigue features found close to the edge of the sample (left) and damage free region closer to the ductile fracture (right)
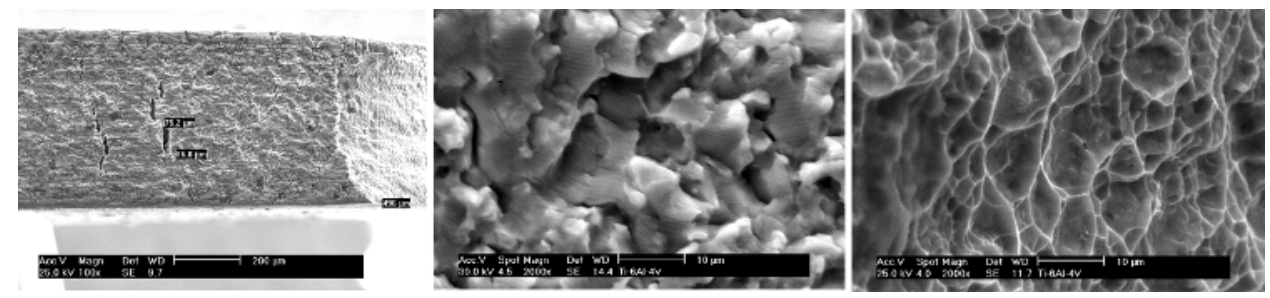

Fig. 7. SEM micrographs of a sample pre-fatigued before tensile testing. Left: microcracks found on the edge of the specimen. Center: fatigue fracture with striations near to the corner where the main crack started. Right: ductile fracture around the middle of the specimen

\section{References}

1. P. Verleysen, J. Degrieck, J.Phys. IV France 134, (2006) 541-546

2. C. Froustey, J.L. Lataillade, Mater. Sci. Eng. A-Struct. Mater. Prop. Microstruct. Process. 500, (2009) 155-163

3. C. Rubio-González, J.A. Gallardo-Gonzĺez, G. Mesmacque, U. Sanchez-Santana, Int.J.Fatigue 30, (2008) 1056-1064

4. Y. Duyi, W. Zhenlin, Mater. Sci. Eng. A-Struct. Mater. Prop. Microstruct. Process. A297, (2001) 54-61

5. ASTM International, ASTM Standard E466-96: Standard Practice for Conducting Force Controlled Constant Amplitude Axial Fatigue Tests of Metallic Materials (1996)

6. ASTM International, ASTM Standard E8M-04: Standard Test Methods for Tension Testing of metallic Materials [Metric] (2004)

7. O. Umezawa, K. Nagai, ISIJ Int. 37(12), (1997) 1170-1179

8. B. Majidi, Metal.Mater.Trans.A 39A, (2008) 772-777 